\title{
Type 1 diabetes mellitus and celiac disease: endothelial dysfunction
}

\author{
Antonio Picarelli · Marco Di Tola · Tania D'Amico • \\ Valeria Mercuri $\cdot$ Patrizia Gargiulo
}

Received: 22 July 2011/ Accepted: 28 July 2011/Published online: 16 September 2011

(C) Springer-Verlag 2011

To the Editor of "Acta Diabetologica".

In the observational study cited above, we evaluated whether the presence of undiagnosed $\mathrm{CD}$ in a group of adult DM1 patients is associated with a different expression of some hemostatic factors, as well as with a different manifestation and/or progression of microvascular complications in comparison with patients suffering from DM1 only. The Ventura group has carefully examined and commented on this study, but we are only partially in agreement with their observations.

First, the including criterion $\mathrm{HbA} 1 \mathrm{c} \leq 7.5 \%$ or $58 \mathrm{mmol} / \mathrm{mol}$ was chosen to ensure the selection of DM1 patients with a satisfactory long-standing metabolic control. This is to prevent that, during the disease progression, an inadequate metabolic control may have affected adversely on the study parameters. The evaluation of hemostatic factors and microvascular complications in DM1 patients also suffering from CD and presenting an unsatisfactory metabolic control could become the object of a future study.

Second, we have chosen to assess the metabolic control by means of HbA1c in order to obtain long-standing information reflecting the chronic state that may culminate in microvascular complications. On the other hand, the metabolic control of adult patients with a long-standing DM1 is generally better than that found in diabetic

\footnotetext{
A. Picarelli $(\bowtie) \cdot$ M. Di Tola

Department of Internal Medicine and Medical Specialities,

Center for Research and Study of Celiac Disease,

Sapienza University, Rome, Italy

e-mail: antonio.picarelli@uniroma1.it

T. D’Amico · V. Mercuri · P. Gargiulo

Endocrinologia "A", Department of Experimental Medicine,

Sapienza University, Rome, Italy
}

children, to whom the Ventura group refers. This is because children have a shorter disease duration and they are more exposed to factors that can alter their metabolic control. In relation to the other parameters able to evaluate the metabolic control, the diabetologists have obviously considered the presence of frequent hypoglycemic events, the extreme glycemia variability, and the increment in daily insulin needs as an implicit exclusion criterion.

Third, in most of the patients evaluated in our study, the presence of other autoimmune diseases has been investigated by means of a wide autoantibody serum panel (data not shown), and the results are not relevant. Finally, since our study was performed on a group of adult DM1 patients, we have obviously not considered anthropometric parameters because the patients enrolled in our study were all already adult, eventually affected by $\mathrm{CD}$, and presenting negative malabsorption clinical picture. It would however be interesting to assess, in the future, the role of $\mathrm{CD}$ on hemostatic factors and microvascular complications also in diabetic children.

In conclusion, we observed a better metabolic control and a thromboresistant condition in adult patients with both DM1 and undiagnosed CD, even if our opinion is that gluten-free diet is mandatory in all patients suffering from CD.

Rome. 22 July 2011. 